\title{
Preface (vol 37, issue 7)
}

\author{
Nadia Magnenat-Thalmann ${ }^{1}$
}

Accepted: 22 May 2021 / Published online: 19 June 2021

(c) The Author(s), under exclusive licence to Springer-Verlag GmbH Germany, part of Springer Nature 2021

The Visual Computer receives more than 1000 submissions a year. Less than $20 \%$ of the submissions are accepted for publication. In addition, the Visual Computer hosts a few special issues, among them the Computer Graphics Conference (CGI) Special Issue. In order to accelerate the publication of the regular accepted papers published online, this issue contains 25 regular papers:

1. Sun Dinghua et al., "Learning wavelet coefficients for face super-resolution"

2. Yongjun He et al., "Overlapping region reconstruction in nuclei image segmentation"

3. Munish Kumar, et al., "Text and graphics segmentation of newspapers printed in Gurmukhi script: a hybrid approach"

4. Aftab Khan, et al., "Arbitrarily shaped Point Spread Function (PSF) estimation for single image blind deblurring"

5. Guoliang Zhang et al., "Auxiliary criterion conversion via spatiotemporal semantic encoding and feature entropy for action recognition"

6. Yuantao Chen et al., "The improved image inpainting algorithm via encoder and similarity constraint"

7. Qian Jiang et al., "Remote sensing image colorization using symmetrical multi-scale DCGAN in YUV color space"

8. Ziheng Zhang et al., "RGB-D-based gaze point estimation via multi-column CNNs and facial landmarks global optimization"

9. Yang Liu et al., "Instance-level 3D shape retrieval from a single image by hybrid-representation-assisted joint embedding"

10. Xingyuan Wang et al., "A new one-dimensional chaotic map and its application in a novel permutation-less image encryption scheme"

11. Jing Zhang et al., "Trajectory and image-based detection and identification of UAV"

Nadia Magnenat-Thalmann

thalmann@miralab.ch

1 MIRALab-CUI, University of Geneva, Battelle, Building A, 7, Route de Drize, 1227 Carouge, Geneva, Switzerland
12. Showmik Bhowmik et al., "Language-invariant novel feature descriptors for handwritten numeral recognition"

13. Wei Guo et al., "Semantic-aware label placement for augmented reality in street view"

14. Saleh Alyat et al., "Two-stream spatiotemporal feature fusion for human action recognition"

15. K. Karthik et al., "A deep neural network model for content-based medical image retrieval with multi-view classification"

16. Cong Wang et al., "Single image deraining via deep shared pyramid network"

17. Qingtang Su et al., "A blind image watermarking scheme combining spatial domain and frequency domain"

18. Juan Villegas-Cortez et al., "Interest points reduction using evolutionary algorithms and CBIR for face recognition"

19. Zhi Han et al., "Low-rank decomposition on transformed feature maps domain for image denoising"

20. Haiyang Meng et al., "Adaptive cropping and deskewing of scanned documents based on high accuracy estimation of skew angle and cropping value"

21. Prashant Goswami et al., "A survey of modeling, rendering and animation of clouds in computer graphics"

22. Sung-Hee Lee et al., "Keyframe-based multi-contact motion synthesis"

23. G. Rohith et al., "Paradigm shifts in super-resolution techniques for remote sensing applications"

24. Chen Luo, et al., "A novel method for reconstructing general 3D curves from stereo images"

25. Hassan Ugail, et al., "A framework for facial age progression and regression using exemplar face templates"

26. Dincer Gokcen, et al., "FPGA-based infrared image deblurring using angular position of IR detector"

Nadia Magnenat-Thalmann.

Editor-in-Chief, the Visual Computer.

Publisher's Note Springer Nature remains neutral with regard to jurisdictional claims in published maps and institutional affiliations. 